\title{
ON THE REGULAR DIGRAPH OF IDEALS OF COMMUTATIVE RINGS
}

\author{
M. AFKHAMI ${ }^{凶}$, M. KARIMI and K. KHASHYARMANESH
}

(Received 23 July 2012; accepted 29 July 2012; first published online 16 October 2012)

\begin{abstract}
Let $R$ be a commutative ring. The regular digraph of ideals of $R$, denoted by $\Gamma(R)$, is a digraph whose vertex set is the set of all nontrivial ideals of $R$ and, for every two distinct vertices $I$ and $J$, there is an arc from $I$ to $J$ whenever $I$ contains a nonzero divisor on $J$. In this paper, we study the connectedness of $\Gamma(R)$. We also completely characterise the diameter of this graph and determine the number of edges in $\Gamma(R)$, whenever $R$ is a finite direct product of fields. Among other things, we prove that $R$ has a finite number of ideals if and only if $\mathrm{N}_{\Gamma(R)}(I)$ is finite, for all vertices $I$ in $\Gamma(R)$, where $\mathrm{N}_{\Gamma(R)}(I)$ is the set of all adjacent vertices to $I$ in $\Gamma(R)$.
\end{abstract}

2010 Mathematics subject classification: primary 05C20; secondary 05C69, 13E05, 16P20.

Keywords and phrases: regular digraph, connectedness, diameter.

\section{Introduction}

The investigation of graphs related to various algebraic structures is a very large and growing area of research. Several classes of graphs associated with algebraic structures have been actively investigated. For example, Cayley graphs have been studied in $[8,11,12,15,18,20]$, power graphs and divisibility graphs have been considered in $[9,10]$, zero-divisor graphs have been studied in $[2-4,6,7]$, and cozerodivisor graphs have been introduced in [1]. Also, comaximal graphs have been studied in $[13,16,19]$.

In [14], Nikmehr and Shaveisi defined the regular digraph of ideals of $R$, denoted by $\overrightarrow{\Gamma_{\text {reg }}}(R)$, as a digraph whose vertex set is the set of all nontrivial ideals of $R$, and, for every two distinct vertices $I$ and $J$, there is an arc from $I$ to $J$, denoted by $I \longrightarrow J$, whenever $I$ contains an element $x$ such that $x y \neq 0$ for all $y \in J$. In other words, $I$ contains a $J$-regular element. They studied and investigated some properties of this graph. For simplicity of notation, we denote this graph by $\Gamma(R)$.

In commutative algebra, regular elements play an important role in the structure of rings (see, for example, [17, Sections 16 and 17]). Thus in this paper we study some more properties of the graph $\Gamma(R)$. In Section 2 we study the connectedness and

(c) 2012 Australian Mathematical Publishing Association Inc. 0004-9727/2012 \$16.00 
diameter of $\Gamma(R)$. Also, we give a very short proof of [14, Theorem 2.1]. Moreover, we generalise [14, Proposition 2.1] and provide necessary and sufficient conditions for connectedness of $\Gamma(R)$, whenever $R$ is an arbitrary commutative ring. Finally, we completely investigate and determine the diameter of $\Gamma(R)$. In Section 3 we determine the isolated vertices in $\Gamma(R)$, and we compute the number of edges in $\Gamma(R)$, whenever $R$ is a finite direct product of fields.

We now recall some definitions and notation on graphs. We use the standard terminology of graphs following [5]. Let $G=(V, E)$ be a simple graph, where $V$ is the set of vertices and $E$ is the set of edges. The graph $H=\left(V_{0}, E_{0}\right)$ is a subgraph of $G$ if $V_{0} \subseteq V$ and $E_{0} \subseteq E$. Moreover, $H$ is called a subgraph induced by $V_{0}$, denoted by $G\left[V_{0}\right]$, if $V_{0} \subseteq V$ and $E_{0}=\left\{\{u, v\} \in E \mid u, v \in V_{0}\right\}$. The distance between two distinct vertices $a$ and $b$ in $G$, denoted by $\mathrm{d}(a, b)$, is the length of the shortest path connecting $a$ and $b$, if such a path exists; otherwise, we set $\mathrm{d}(a, b):=\infty$. The diameter of a graph $G$ is denoted by $\operatorname{diam}(G)$ and is defined as the supremum of the set of all distances $\mathrm{d}(a, b)$ for all pairs $(a, b)$, where $a$ and $b$ are distinct vertices of $G$. Also, for two distinct vertices $a$ and $b$ in $G$, the notation $a-b$ means that $a$ and $b$ are adjacent. A graph $G$ is said to be connected if there exists a path between any two distinct vertices, and it is complete if it is connected with diameter one. We use $K_{n}$ to denote the complete graph with $n$ vertices. We say that $G$ is empty if no two vertices of $G$ are adjacent. For a vertex $x$ in $G$, we denote the set of all vertices adjacent to $x$ by $\mathrm{N}_{G}(x)$, and the size of $\mathrm{N}_{G}(x)$ is called the degree of $x$ in $G$, denoted by $\operatorname{deg}(x)$. A vertex $x$ is isolated, if $\mathrm{N}_{G}(x)=\emptyset$.

Let $\Gamma$ be a digraph. An arc from a vertex $x$ to another vertex $y$ of $\Gamma$ is denoted by $x \rightarrow y$. We say that $\Gamma$ is weakly connected if the undirected underlying simple graph obtained by replacing all directed edges of $\Gamma$ with undirected edges is a connected graph. Also, the in-degree (out-degree) of a vertex $x$ in a digraph $G$ is the number of arcs to (away from) $x$ which is denoted by $\mathrm{d}^{+}(x)\left(\mathrm{d}^{-}(x)\right)$.

Throughout this paper, all rings are assumed to be commutative with identity. We denote by $\operatorname{Max}(R)$ and $\operatorname{Nil}(R)$ the set of all maximal ideals and the set of all nilpotent elements of $R$, respectively. Also, the set of all zero-divisors of an $R$-module $M$, which is denoted by $Z(M)$, is the set

$$
Z(M)=\{r \in R \mid r x=0 \text {, for some nonzero element } x \text { in } M\} .
$$

An element $r \in R$ is called $M$-regular if $r \notin Z(M)$. An $R$-sequence is a $d$-tuple $r_{1}, \ldots, r_{d}$ in $R$ such that, for every $i \leq d, r_{i}$ is $R /\left(r_{1}, r_{2}, \ldots, r_{i-1}\right)$-regular. We say that $\operatorname{depth}(R)=0$, whenever every nonunit element of $R$ is a zero-divisor.

\section{Connectedness of $\Gamma(R)$}

In this section we study the weak connectedness of $\Gamma(R)$. We also completely characterise the diameter of $\Gamma(R)$.

For an arbitrary element $r \in R$, we set

$$
\operatorname{Ann}(r)=\operatorname{Ann}(r R)=\{s \in R \mid s r=0\} .
$$


Also, for an ideal $I$ of $R$ we put $\operatorname{Ann}(I)=\bigcap_{s \in I} \operatorname{Ann}(s)$. Moreover, the set of all associated prime ideals of $R$ is defined as follows: $\operatorname{Ass}(R)=\{\mathfrak{p} \mid \mathfrak{p}$ is a prime ideal of $R$ and there exists $r \in R$ such that $\mathfrak{p}=\operatorname{Ann}(r)\}$.

Remark 2.1. If $R$ is Noetherian and $\operatorname{depth}(R)=0$, then $R$ contains a finite number of maximal ideals and $\operatorname{Ann}(\mathrm{m}) \neq 0$, for all maximal ideals $\mathrm{m}$ in $R$.

The following lemma is needed in the rest of the paper.

Lemma 2.2. Let $R$ be a ring and $\mathrm{m}$ be a maximal ideal in $R$. If $\mathrm{Ann}(\mathrm{m}) \neq 0$, then $\mathrm{m}=\mathrm{Z}(\operatorname{Ann}(\mathrm{m}))$.

Proof. Since $m \operatorname{Ann}(m)=0$, we have $m \subseteq Z(\operatorname{Ann}(m))$. Now, suppose that $x$ is an arbitrary element in $\mathrm{Z}(\mathrm{Ann}(\mathrm{m}))$. Then there is a nonzero element $y \in \operatorname{Ann}(\mathrm{m})$ such that $x y=0$. Assume to the contrary that $x \notin m$. So $y \in \mathfrak{m}$ and $R x+\mathfrak{m}=R$. Therefore $r x+h=1$, for some $r \in R$ and $h \in \mathrm{m}$, and hence $r x y+h y=y$. Now, since $x y=0=y h$, we have that $y=0$, which is a contradiction.

The following corollary immediately follows from Lemma 2.2.

Corollary 2.3. Suppose that $R$ is Noetherian with $\operatorname{depth}(R)=0$ and $\operatorname{Max}(R)=$ $\left\{\mathrm{m}_{1}, \ldots, \mathrm{m}_{n}\right\}$. Then we have the following statements.

(i) If $n \geq 2$, then $\mathrm{m}_{i} \longrightarrow \operatorname{Ann}\left(\mathrm{m}_{j}\right)$ in $\Gamma(R)$, for all $i \neq j$.

(ii) $\mathrm{Z}\left(\sum_{i=1}^{n} \operatorname{Ann}\left(\mathrm{m}_{i}\right)\right)=\mathrm{Z}(R)$.

Lemma 2.4. Assume that $R$ is a Noetherian ring with $\operatorname{depth}(R)=0$. Put $n:=|\operatorname{Max}(R)|$. Then we have the following statements.

(i) If $\operatorname{Ann}\left(\mathrm{m}_{i}\right) \subseteq \mathrm{m}_{i}$, for all $1 \leq i \leq n$, then $\mathrm{Z}(\mathrm{Nil}(R))=\mathrm{Z}(R)$.

(ii) If $R$ is reduced, then $R$ is a finite direct product of fields.

Proof. (i) Since $m_{i} \operatorname{Ann}\left(m_{i}\right)=0$ and $\operatorname{Ann}\left(m_{i}\right) \subseteq \mathfrak{m}_{i}$, for all $1 \leq i \leq n$, we have $\operatorname{Ann}\left(\mathfrak{m}_{i}\right) \subseteq \bigcap_{\mathfrak{p} \in \operatorname{Min}(R)} \mathfrak{p}$, for $i=1, \ldots, n$. Hence $\sum_{i=1}^{n} \operatorname{Ann}\left(\mathfrak{m}_{i}\right) \subseteq \operatorname{Nil}(R)$. Now the result follows from Corollary 2.3(ii).

(ii) If $(R, \mathfrak{m})$ is a local ring, then clearly $\operatorname{Ann}(\mathfrak{m}) \subseteq \operatorname{Nil}(R)$. If $m \neq 0$, then $\operatorname{Ann}(\mathfrak{m}) \neq$ 0 , which implies that $\operatorname{Nil}(R) \neq 0$. This violates our assumption. Therefore $m=0$, and so $R$ is a field. Now suppose that $R$ is not local. Then, by (i), there exists a maximal ideal $\mathfrak{m}_{i}$ such that $\operatorname{Ann}\left(\mathfrak{m}_{i}\right) \nsubseteq \mathfrak{m}_{i}$. Thus $R \cong R / \mathfrak{m}_{i} \times R / \operatorname{Ann}\left(\mathfrak{m}_{i}\right)$. Now $R / \operatorname{Ann}\left(\mathfrak{m}_{i}\right)$ is also reduced, $\operatorname{depth}\left(R / \operatorname{Ann}\left(\mathrm{m}_{i}\right)\right)=0$, and $R / \operatorname{Ann}\left(\mathrm{m}_{i}\right)$ has $n-1$ maximal ideals. Now, by using induction on $n$, the result holds.

In the following theorem, we provide a very short proof of [14, Theorem 2.1].

Theorem 2.5. Let $R$ be a Noetherian ring. Then $\Gamma(R)$ is an empty graph if and only if $R$ is either an Artinian local ring or a direct product of two fields.

Proof. First suppose that $\Gamma(R)$ is an empty graph. If $R$ contains a regular element, then $\Gamma(R)$ is a refinement of a star graph, which is impossible. So we have that $\operatorname{depth}(R)=0$. 
If $R$ is a local ring with a maximal ideal $m$ and $\mathfrak{p}$ is a minimal prime ideal of $R$, then $\mathfrak{p} \in \operatorname{Ass}(R)$, and so $\mathfrak{p}=\operatorname{Ann}(x)$, for some $x \in R$. Hence $R / \mathfrak{p} \cong R x$ and $\mathrm{Z}(R x)=\mathfrak{p}$. Now since $\Gamma(R)$ is an empty graph, we have that $\mathrm{Z}(I)=\mathrm{m}$, for all nontrivial ideals $I$ in $R$. In particular we have $\mathfrak{p}=\mathrm{Z}(R x)=\mathfrak{m}$, and this implies that $R$ is Artinian. Hence, in this situation, $R$ is an Artinian local ring. Now, if $R$ is not local, then there exist two distinct maximal ideals $m_{1}$ and $m_{2}$. Since $\Gamma(R)$ is empty, by Corollary 2.3 , we have $\operatorname{Ann}\left(m_{1}\right)=m_{2}$. Also we have $m_{1}+m_{2}=R$. Therefore we can easily see that $R$ is a direct product of two fields.

Conversely, assume that $R$ is either an Artinian local ring or a direct product of two fields. Firstly suppose that $R$ is an Artinian local ring with the maximal ideal $m$. Since $\operatorname{Ass}(R)=\{\mathfrak{m}\}$, we have that $\mathrm{Z}(I)=\mathrm{Z}(J)=\mathfrak{m}$, for all ideals $I, J$ of $R$. This means that $\Gamma(R)$ is empty. Also if $R$ is a direct product of two fields, then $\Gamma(R) \cong 2 \overline{K_{2}}$.

Lemma 2.6. Let $R$ be a nonreduced ring. Then $\mathrm{Nil}(R) \subseteq \mathrm{Z}(I)$, for all nontrivial ideals I of $R$.

Proof. Assume to the contrary that $\operatorname{Nil}(R) \nsubseteq \mathrm{Z}(I)$, for some nontrivial ideal $I$ of $R$. Thus there is a nonzero element $x$ in $\operatorname{Nil}(R)$ such that $x y \neq 0$, for all nonzero elements $y \in I$. Hence $x^{2} y=x(x y) \neq 0$, and so we can easily see that $x^{n} y \neq 0$, for all positive integers $n$. But since $x^{k}=0$, for some $k \geq 2$, we have that $x^{k} y=0$, which is a contradiction.

Lemma 2.7. Let $R$ be a Noetherian ring. Then $R$ is an Artinian local ring if and only if the graph $\Gamma(F \times R)$ is disconnected, where $F$ is a field.

Proof. First suppose that $R$ is an Artinian local ring. If $R$ is a field, then, by Theorem 2.5, $\Gamma(F \times R)$ is an empty graph. So we may assume that $R$ is not a field. Then, for any nontrivial ideal $I$ of $R$, the element $(1,0) \in F \times I$ is $(F \times 0)$-regular, and so we have that $F \times I \longrightarrow F \times 0$ in $\Gamma(R)$. Hence the induced subgraph of $\Gamma(R)$ with vertex set $A=\{F \times 0, F \times I \mid I$ is a nontrivial ideal of $R\}$ is a star graph. Also $(0,1) \in 0 \times R$ is a $(0 \times J)$-regular element, for all nontrivial ideals $J$ of $R$, and hence $0 \times R \longrightarrow 0 \times J$ in $\Gamma(R)$. This implies that the induced subgraph of $\Gamma(R)$ with vertex set $B=\{0 \times R, 0 \times J \mid J$ is a nontrivial ideal of $\mathrm{R}\}$ is a star graph. Now, by Theorem 2.5, it is easy to see that $\Gamma(F \times R)$ is disconnected with connected components $A$ and $B$.

Conversely, suppose that $\Gamma(F \times R)$ is disconnected. Assume to the contrary that $R$ is not an Artinian local ring. If $R$ is a direct product of two fields, then $\Gamma(F \times R) \cong C_{6}$ (see [14, Proposition. 3.6]), which is impossible. So $R$ is not a direct product of two fields. Now, by Theorem 2.5, we have an edge $I \longrightarrow J$ in $\Gamma(R)$. Let $A$ and $B$ be the sets as defined in the first paragraph in this proof. Then the edge $F \times I \longrightarrow 0 \times J$ connects the sets $A$ and $B$. Thus $\Gamma(R)$ is connected, which is a contradiction.

In [14, Proposition 2.1], the authors establish a result on the connectedness of $\Gamma(R)$ for Noetherian local rings. In the following theorem, we generalise [14, Proposition 2.1] and provide necessary and sufficient conditions for connectedness of $\Gamma(R)$, where $R$ is an arbitrary commutative ring. 
Theorem 2.8. Let $R$ be a Noetherian ring. The graph $\Gamma(R)$ is connected if and only if one of the following statements holds.

(i) $\operatorname{depth}(R) \neq 0$.

(ii) $\operatorname{depth}(R)=0$ and $R=F \times R^{\prime}$, where $F$ is a field and $R^{\prime}$ is not an Artinian local ring.

Proof. Suppose that $\Gamma(R)$ is connected and $\operatorname{depth}(R)=0$. If $\operatorname{Ann}(\mathrm{m}) \subseteq \mathrm{m}$, for all maximal ideals $\mathrm{m}$, then, by Lemma 2.4, we have $\mathrm{Z}(\mathrm{Nil}(R))=\mathrm{Z}(R)$. Also, in view of Lemma 2.6, $\operatorname{Nil}(R) \neq 0$ is an isolated vertex which implies that $\Gamma(R)$ is disconnected. So there exists a maximal ideal $m$ such that $\operatorname{Ann}(\mathfrak{m}) \nsubseteq \mathfrak{m}$. Hence $\operatorname{Ann}(\mathfrak{m})+\mathfrak{m}=R$. Also, since $m \operatorname{Ann}(m)=0$, we have that $R=R / m \times R / A n n(m)$. Moreover, by Lemma 2.7, $R$ is not an Artinian local ring.

Conversely, assume that one of the conditions (i) or (ii) is satisfied. Condition (i) implies that $\Gamma(R)$ is a refinement of a star graph, and so it is connected. If (ii) is satisfied, then, by Lemma 2.7 , the result holds.

According to Theorem 2.8, $\Gamma(R)$ has isolated vertices if $\Gamma(R)$ is disconnected and $R$ is indecomposable. We denote the number of nonsingular connected components of $\Gamma(R)$ by $\pi(R)$. In the next theorem we compute $\pi(R)$.

Theorem 2.9. Let $R$ be a Noetherian ring. Suppose that $\Gamma(R)$ is disconnected and $\operatorname{depth}(R)=0$. Then the following statements hold.

(i) $\quad$ If $|\operatorname{Max}(R)| \geq 3$, or $|\operatorname{Max}(R)|=2$ and $R$ is not Artinian, then $\pi(R)=1$.

(ii) If $R=R_{1} \times R_{2}$, where $R_{1}, R_{2}$ are two Artinian local rings which are not both fields, then $\pi(R)=2$.

(iii) If $R=R_{1} \times R_{2}$, where $R_{1}, R_{2}$ are two fields, or $R$ is a local Artinian ring, then $\pi(R)=0$.

Proof. (i) Suppose that $|\operatorname{Max}(R)| \geq 3$. Clearly, for each arc $I \longrightarrow J$ in $\Gamma(R)$, we have $\mathrm{m} \longrightarrow J$, for all maximal ideals $m$ containing $I$. Hence, if $C$ is a nonsingular connected component of $\Gamma(R)$, then it contains a maximal ideal. Thus it is enough to show that there is a path joining any two maximal ideals of $R$. To this end, suppose that $\mathrm{m}_{1}, \mathrm{~m}_{2}$ are arbitrary maximal ideals and $m_{3}$ is a maximal ideal distinct from $m_{1}, m_{2}$ in $R$. By Corollary 2.3 , we have the path $m_{1} \longrightarrow \operatorname{Ann}\left(m_{3}\right) \longleftarrow m_{2}$. Thus all maximal ideals lie in the same component, which implies that $\pi(R)=1$.

Now suppose that $\operatorname{Max}(R)=\{\mathfrak{m}, \mathfrak{n}\}$ and $R$ is not Artinian. We claim that $\operatorname{Ann}(\mathfrak{m}) \subseteq$ $\mathrm{m}$ and $\operatorname{Ann}(\mathfrak{n}) \subseteq \mathfrak{n}$. Assume to the contrary that $\operatorname{Ann}(\mathrm{m}) \nsubseteq \mathrm{m}$. Then we have $R=$ $R / \mathfrak{m} \times R / \operatorname{Ann}(\mathfrak{m})$. Since $|\operatorname{Max}(R)|=2$, the ring $R / \operatorname{Ann}(\mathfrak{m})$ is local. Now, since $\Gamma(R)$ is disconnected, by Theorem 2.8, we have that $R / \operatorname{Ann}(\mathrm{m})$ is $\operatorname{Artinian}$, and so $R$ is Artinian, which is the required contradiction. Now, by Lemma 2.4, it is easy to see that $\mathrm{Z}(\mathrm{m} \cap \mathfrak{n})=\mathrm{Z}(R)$. This means that $\mathrm{d}^{+}(\mathrm{m} \cap \mathfrak{n})=0$. Hence if $\mathrm{m} \cap \mathfrak{n}$ is an isolated

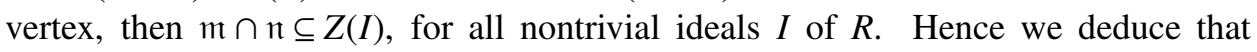
$\mathrm{m} \cap \mathfrak{n}=\operatorname{Nil}(R)$, and so $R$ is an Artinian ring, which is impossible. Hence $\mathrm{m} \cap \mathfrak{n} \longrightarrow J$, for some nontrivial ideal $J$ of $R$. Now consider the path $\mathrm{m} \longrightarrow J \longleftarrow n$ to deduce that $\mathrm{m}$ and $\mathrm{n}$ lie in the same component, and hence $\pi(R)=1$. 
(ii) Suppose that $R=R_{1} \times R_{2}$, where $R_{1}, R_{2}$ are two Artinian local rings at least one of which is not a field. Without loss of generality, we may assume that $R_{1}$ is not a field. Consider two sets of edges

$$
A=\left\{R_{1} \times J \longrightarrow I \times 0\right\}_{J \neq R_{2}, I \neq 0}
$$

and

$$
B=\left\{0 \times J \longleftarrow I \times R_{2}\right\}_{J \neq 0, I \neq R_{1}} .
$$

It is easy to see that $\Gamma(R)$ has only two nonsingular connected components $A$ and $B$. Note that all other parts of $\Gamma(R)$ are isolated vertices. Thus $\pi(R)=2$.

(iii) By Theorem 2.5, the result holds.

For any two subsets $A$ and $B$ of vertices in $\Gamma(R)$, we use the notation $\mathrm{d}(A, B)$ to denote the maximum distance between vertices in $A$ and vertices in $B$.

Theorem 2.10. Let $R$ be a Noetherian ring such that $\Gamma(R)$ is connected. Then the following statements hold.

(i) $\operatorname{diam}(\Gamma(R))=1$ if and only if $R$ is an integral domain.

(ii) $\operatorname{diam}(\Gamma(R))=2$ if and only if $R$ is not an integral domain and $\operatorname{depth}(R) \neq 0$.

(iii) If $\operatorname{depth}(R)=0$, then we have the following statements.

(a) $\operatorname{diam}(\Gamma(R))=3$ if and only if $R=F_{1} \times F_{2} \times R_{2}$, where $F_{1}$ and $F_{2}$ are fields and $\mathrm{Z}\left(\mathrm{Nil}\left(R_{2}\right)\right) \neq \mathrm{Z}\left(R_{2}\right)$.

(b) $\operatorname{diam}(\Gamma(R))=4$ if and only if $R=F_{1} \times F_{2} \times R_{2}$, where $F_{1}$ and $F_{2}$ are fields and $\mathrm{Z}\left(\mathrm{Nil}\left(R_{2}\right)\right)=\mathrm{Z}\left(R_{2}\right)$.

(c) $\operatorname{diam}(\Gamma(R))=5$ if and only if $R$ is non of the rings as in $(a)$ and $(b)$.

Proof. (i) The result follows by [14, Thoerem 3.1].

(ii) Firstly, suppose that $R$ is not an integral domain and $\operatorname{depth}(R) \neq 0$. Thus clearly $\Gamma(R)$ is a refinement of a star graph, and so $\operatorname{diam}(\Gamma(R))=2$.

Conversely, suppose that $\operatorname{diam}(\Gamma(R))=2$. By part (i), $R$ is not an integral domain. Now assume to the contrary that $\operatorname{depth}(R)=0$. Since $\Gamma(R)$ is connected, by Theorem 2.8, we have $R=F_{1} \times R_{1}$, where $F_{1}$ is a field and $R_{1}$ is a commutative ring which is not an Artinian local ring. Then it is easy to see that $\mathrm{d}\left(F_{1} \times 0,0 \times R_{1}\right)=3$, which is a contradiction.

(iii) Since $\Gamma(R)$ is connected, by Theorem 2.8, we have that $R=F_{1} \times R_{1}$, where $F_{1}$ is a field and $F_{1}$ is not an Artinian local ring. We have two cases to consider.

Case 1. If there exists a field $F_{2}$ such that $R_{1}=F_{2} \times R_{2}$ for some nonzero commutative ring $R_{2}$, then we claim that $\operatorname{diam}(\Gamma(R))=3$ or 4 . Whenever $R_{2}$ is a field, then as we mentioned in the proof of Lemma 2.7, the graph $\Gamma\left(F_{1} \times F_{2} \times R_{2}\right)$ is isomorphic to $C_{6}$. So we may assume that $R_{2}$ is not a field. Now, we consider the following partition for vertices of $\Gamma(R)$ :

$C_{1}:=\left\{F_{1} \times F_{2} \times 0, F_{1} \times 0 \times 0,0 \times F_{2} \times 0, F_{1} \times F_{2} \times I \mid I\right.$ is a nontrivial ideal of $\left.R_{2}\right\}$, $C_{2}:=\left\{0 \times 0 \times R_{2}, F_{1} \times 0 \times R_{2}, 0 \times F_{2} \times R_{2}, 0 \times 0 \times I \mid I\right.$ is a nontrivial ideal of $\left.R_{2}\right\}$, 


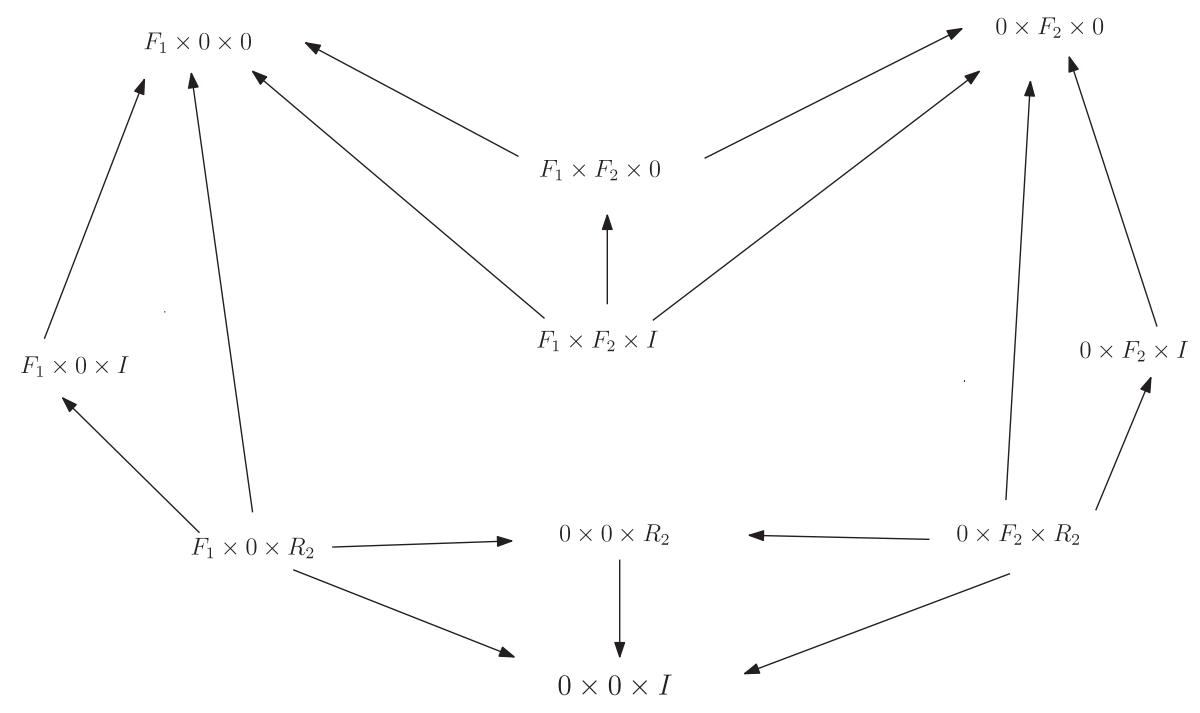

Figure 1. A part of $\Gamma\left(F_{1} \times F_{2} \times R_{2}\right)$.

$C_{3}:=\left\{F_{1} \times 0 \times I \mid I\right.$ is a nontrivial ideal of $\left.R_{2}\right\}$,

$C_{4}:=\left\{0 \times F_{2} \times I \mid I\right.$ is a nontrivial ideal of $\left.R_{2}\right\}$.

We need only determine $\mathrm{d}\left(C_{i}, C_{j}\right)$ for $1 \leq i, j \leq 4$. For an arbitrary nontrivial ideal $I$ of $R_{2}$, consider Figure 1, which shows some parts of $\Gamma(R)$. Now, it is routine to check that

$$
\mathrm{d}\left(C_{i}, C_{j}\right)= \begin{cases}1 \text { or } 2 & \text { for } i=j=1,2,3,4 \\ 3 \text { or } 4 & \text { for } i=3 \text { and } j=4 \\ 1 \text { or } 2 \text { or } 3 & \text { otherwise. }\end{cases}
$$

This implies that whenever $\mathrm{d}\left(C_{3}, C_{4}\right)=4$, then $\operatorname{diam}(\Gamma(R))=4$. Otherwise $\operatorname{diam}(\Gamma(R))=3$. So we need only consider the situations in which $\operatorname{diam}(\Gamma(R))=4$. We claim that $\operatorname{diam}(\Gamma(R))=4$ if and only if $\Gamma\left(R_{2}\right)$ has an isolated vertex and $R_{2}$ is not reduced. To prove the claim we consider the following situations.

$(\alpha) \Gamma\left(R_{2}\right)$ has no isolated vertex. Let $F_{1} \times 0 \times I$ and $0 \times F_{2} \times J$ be arbitrary vertices in $C_{3}$ and $C_{4}$, respectively. Since $I$ is not an isolated vertex in $\Gamma\left(R_{2}\right)$, there exists an ideal $I^{\prime}$ of $R_{2}$ such that $I \longrightarrow I^{\prime}$ or $I^{\prime} \longrightarrow I$ in $\Gamma\left(R_{2}\right)$. Hence we have the paths

$$
F_{1} \times 0 \times I \longrightarrow 0 \times 0 \times I^{\prime} \longleftarrow 0 \times F_{2} \times R_{2} \longrightarrow 0 \times F_{2} \times J
$$

or

$$
F_{1} \times 0 \times I \longleftarrow F_{1} \times F_{2} \times I^{\prime} \longrightarrow 0 \times F_{2} \times 0 \longleftarrow 0 \times F_{2} \times J .
$$

These imply that $\mathrm{d}\left(C_{3}, C_{4}\right)=3$.

$(\boldsymbol{\beta}) R_{2}$ is a reduced ring. Since $\operatorname{depth}(R)=0$, we have that $\operatorname{depth}\left(R_{2}\right)=0$. Now, in view of Lemma 2.4(ii), $R_{2}$ is a finite direct product of fields $F_{1}^{\prime}, \ldots, F_{n}^{\prime}$. Since $R_{2}$ is not a field, $n \geq 2$. If $n=2$, then $\mathrm{V}\left(\Gamma\left(R_{2}\right)\right)=\left\{F_{1}^{\prime} \times 0,0 \times F_{2}^{\prime}\right\}$. Now if $I$ and $J$ are vertices 


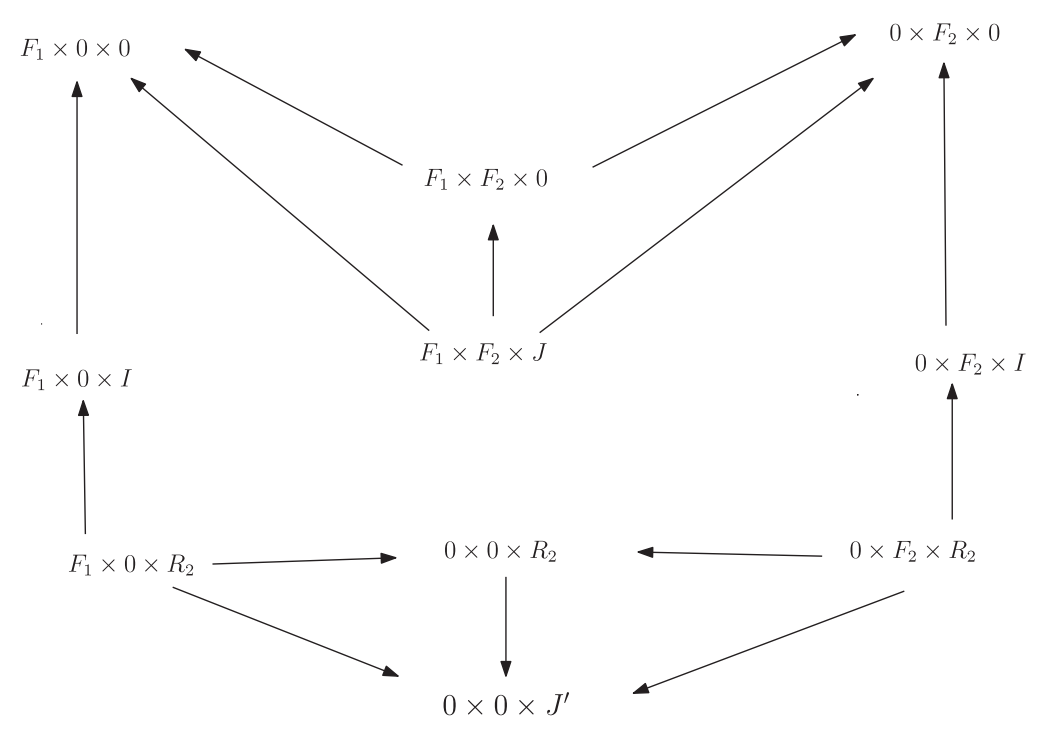

FIGURE 2. A part of $\Gamma\left(F_{1} \times F_{2} \times R_{1}\right)$ where $J^{\prime} \neq 0$ and $J \neq R_{2}$.

in $\Gamma\left(R_{2}\right)$, then we have the following path in $\Gamma(R)$ :

$$
F_{1} \times 0 \times I \longleftarrow F_{1} \times F_{2} \times I \longrightarrow 0 \times F_{2} \times 0 \longleftarrow 0 \times F_{2} \times J .
$$

This means that $\mathrm{d}\left(C_{3}, C_{4}\right)=3$. Also, whenever $n \geq 3$, by Theorem 2.8(ii), $\Gamma\left(R_{2}\right)$ is connected, and so it has no isolated vertices. Thus, by $(\alpha)$, we have that $\mathrm{d}\left(C_{3}, C_{4}\right)=3$. $(\gamma) R_{2}$ is not reduced and there exists an isolated vertex $I$ in $\Gamma\left(R_{2}\right)$. Now consider the vertices $F_{1} \times 0 \times I$ and $0 \times F_{2} \times I$ in $\Gamma(R)$. It follows from Figure 2 that $\mathrm{d}\left(F_{1} \times 0 \times I\right.$, $\left.0 \times F_{2} \times I\right)=4$. This implies that $\mathrm{d}\left(C_{3}, C_{4}\right)=4$.

Case 2. $R_{1}$ is indecomposable or $R_{1}$ has no decomposition $R_{1}=F_{2} \times R_{2}$, for some field $F_{2}$. Now let $m$ be an arbitrary maximal ideal of $R_{1}$. If $\operatorname{Ann}(\mathfrak{m}) \nsubseteq \mathfrak{m}$, then $\operatorname{Ann}(\mathrm{m})$ and $\mathrm{m}$ are comaximal, and so $R_{1}=R_{1} / \mathrm{m} \times R_{1} / \mathrm{Ann}(\mathrm{m})$ which is impossible. Hence $\operatorname{Ann}(\mathrm{m}) \subseteq \mathfrak{m}$ for all maximal ideals $\mathrm{m}$ of $R_{1}$. Now, by Lemmas 2.4 and 2.6, $\operatorname{Nil}\left(R_{1}\right)$ is an isolated vertex in $\Gamma\left(R_{1}\right)$. Thus

$$
\mathrm{d}^{-}\left(F_{1} \times \operatorname{Nil}\left(R_{1}\right)\right)=\mathrm{d}^{+}\left(0 \times \operatorname{Nil}\left(R_{1}\right)\right)=1
$$

and

$$
\mathrm{d}^{+}\left(F_{1} \times \operatorname{Nil}\left(R_{1}\right)\right)=\mathrm{d}^{-}\left(0 \times \operatorname{Nil}\left(R_{1}\right)\right)=0 .
$$

On the other hand, since the graph $\Gamma\left(F_{1} \times R_{1}\right)$ is connected, there exists an arc $F_{1} \times I \longrightarrow 0 \times J$ for some ideals $I$ and $J$ of $R_{1}$. Hence there exists a path (with minimum length)

$$
F_{1} \times \operatorname{Nil}\left(R_{1}\right) \longrightarrow F_{1} \times 0 \longleftarrow F_{1} \times I \longrightarrow 0 \times J \longleftarrow 0 \times R_{1} \longrightarrow 0 \times \operatorname{Nil}\left(R_{1}\right)
$$

in $\Gamma(R)$. Therefore in this situation we have $\operatorname{diam}(\Gamma(R))=5$. 


\section{Degrees of the vertices and counting the edges}

We begin this section with the following proposition which determines the isolated vertices in $\Gamma(R)$.

Proposition 3.1. Let $R$ be a nonreduced Noetherian ring with $\operatorname{depth}(R)=0$. Then $I$ is an isolated vertex in $\Gamma(R)$ if and only if $I$ is a nilpotent ideal and $\mathrm{Z}(I)=\mathrm{Z}(R)$.

Proof. Let $I$ be an isolated vertex in $\Gamma(R)$. We claim that $I \subseteq \mathrm{Z}(I)$. Assume to the contrary that $I \nsubseteq \mathrm{Z}(I)$. We consider the following cases.

Case 1. There exists an ideal $J$ of $R$ such that $I \subset J$. Thus $J \nsubseteq \mathrm{Z}(I)$. Hence there exists an $\operatorname{arc} J \longrightarrow I$ in $\Gamma(R)$. Since $I$ is an isolated vertex, this is impossible.

Case 2. There exists an ideal $K$ of $R$ such that $K \subset I$. By using a method similar to that used in Case $1, K \longrightarrow I$, which again is impossible.

Now it follows from the above cases that $I$ is both a minimal and maximal ideal of $R$. Hence $\operatorname{Ann}(I)$ is a maximal ideal of $R$, and so $R \cong R / I \times R / \operatorname{Ann}(I)$. This implies that $R$ is reduced, which is the required contradiction. Thus $I \subseteq \mathrm{Z}(I)$. Now it follows from our claim that $I \subseteq \mathrm{Z}(J)$ for all ideal $J$ of $R$. Hence $I \subseteq \bigcap_{J \unlhd R} \mathrm{Z}(J)$. Moreover, for any minimal prime ideal $\mathfrak{p}$ of $R$, there exists an element $x$ in $R$ such that $\mathfrak{p}=\operatorname{Ann}(x)$. Thus $\mathfrak{p}=\mathrm{Z}(R x)$. Hence $I \subseteq \mathfrak{p}$, and so $I \subseteq \operatorname{Nil}(R)$. Also it follows from our claim that $\mathrm{m} \subseteq \mathrm{Z}(I)$ for all maximal ideals $\mathrm{m}$ of $R$. Hence $\bigcup_{\mathrm{m} \in \operatorname{Max}(R)} \mathrm{m} \subseteq \mathrm{Z}(I)$, which implies that $\mathrm{Z}(R)=\mathrm{Z}(I)$.

The converse implication follows from Lemma 2.6

COROLlaRy 3.2. If $R$ is a nonreduced ring such that $\Gamma(R)$ contains an isolated vertex, then $\operatorname{Nil}(R)$ is an isolated vertex in $\Gamma(R)$.

Proof. Suppose that $I$ is an isolated vertex in $\Gamma(R)$. Then, in view of Proposition 3.1, $I \subseteq \operatorname{Nil}(R)$ and $\mathrm{Z}(I)=\mathrm{Z}(R)$. This implies that $\mathrm{Z}(\mathrm{Nil}(R))=\mathrm{Z}(R)$. Again, by Proposition 3.1, $\operatorname{Nil}(R)$ is an isolated vertex in $\Gamma(R)$.

Theorem 3.3. Suppose that $R$ is a Noetherian ring such that $\operatorname{depth}(R)=0$ and that the graph $\Gamma(R)$ is not empty. Then the following conditions are equivalent.

(i) $R$ has a finite number of ideals.

(ii) $\mathrm{N}_{\Gamma(R)}(I)$ is finite, for all vertices $I$ in $\Gamma(R)$.

(iii) $V(\Gamma(R))-\mathrm{N}_{\Gamma(R)}(I)$ is finite, for all vertices $I$ in $\Gamma(R)$.

Proof. The implications (i) $\Longrightarrow$ (ii) and (i) $\Longrightarrow$ (iii) are trivial.

(ii) $\Longrightarrow$ (i) First note that if $R$ is reduced, then, by Lemma 2.4(ii), $R$ is a finite direct product of fields, and so it has a finite number of ideals. So we may assume that $R$ is not reduced.

Now we claim that $R$ has a decomposition as $R \cong R_{1} \times R_{2}$ for some nonzero rings $R_{1}$ and $R_{2}$. We consider two cases.

Case 1. There exists a maximal ideal $m$ of $R$ such that $\operatorname{Ann}(\mathfrak{m}) \nsubseteq \mathfrak{m}$. This implies that $R$ has the decomposition $R \cong R / \operatorname{Ann}(\mathrm{m}) \times R / \mathrm{m}$. 
Case 2. For all maximal ideals $m$ of $R$, Ann $(\mathfrak{m}) \subseteq \mathfrak{m}$. Hence, by Lemma 2.4, $\mathrm{Z}(\operatorname{Nil}(R))=\mathrm{Z}(R)$. Also, since $\operatorname{Nil}(R) \subseteq \mathrm{J}(R)$, we have that $\mathrm{Z}(\mathrm{J}(R))=\mathrm{Z}(R)$, and so, for any ideal $I$ of $R$, we have no arc $I \longrightarrow \mathrm{J}(R)$ in $\Gamma(R)$. Now we show that $\mathrm{J}(R)$ is an isolated vertex in $\Gamma(R)$. To achieve this, assume to the contrary that $\mathrm{J}(R)$ is not an isolated vertex. Hence there exists an $\operatorname{arc} \mathrm{J}(R) \rightarrow I$ in $\Gamma(R)$, for some ideal $I$ of $R$. Clearly $I \mathrm{~J}(R)^{i} \neq 0$ for all $i \geq 0$. Moreover, by Nakayama's lemma, $I \mathrm{~J}(R)^{i} \neq I \mathrm{~J}(R)^{j}$ for all $i \neq j$. But $\mathrm{J}(R) \longrightarrow I \mathrm{~J}(R)^{i}$ for all $i>0$, which contradicts (ii). Thus $\mathrm{J}(R)$ is an isolated vertex, and so, by Proposition 3.1, $\mathrm{J}(R)$ is nilpotent. This implies that the ring $R$ is Artinian, and so $R \cong R_{1} \times R_{2}$ for some nonzero rings $R_{1}$ and $R_{2}$. Now, clearly, for any ideal $I$ of $R_{1}$, there exists an arc $R_{1} \times 0 \longrightarrow I \times 0$ in $\Gamma(R)$. So, by using assumption (ii), $R_{1}$ has a finite number of ideals. Similarly $R_{2}$ has a finite number of ideals. This implies that the ring $R$ has finite number of ideals.

(iii) $\Longrightarrow$ (i) Again we may assume that $R$ is not reduced. We have two cases.

Case $1^{\prime}$. There exists a maximal ideal $\mathrm{m}$ of $R$ such that Ann $(\mathrm{m}) \nsubseteq \mathrm{m}$. Then $R \cong R_{1} \times R_{2}$ for some nonzero rings $R_{1}$ and $R_{2}$. Now, for any ideal $K$ of $R_{2}$, there is no adjacency between two vertices $R_{1} \times 0$ and $0 \times K$. Hence (iii) implies that $R_{2}$ has a finite number of ideals. Similarly, $R_{1}$ also has a finite number of ideals, and so (i) is proved.

Case $2^{\prime}$. For all maximal ideals $m$ of $R, \operatorname{Ann}(\mathfrak{m}) \subseteq \mathfrak{m}$. Again, by Lemma 2.4, $\mathrm{Z}(\operatorname{Nil}(R))=\mathrm{Z}(R)$. Hence, in view of Proposition 3.1, $\mathrm{Nil}(R)$ is an isolated vertex in $\Gamma(R)$. Thus, by (iii), $R$ has only a finite number of ideals.

Corollary 3.4. The graph $\Gamma(R)$ is finite if and only if $R$ has a finite number of ideals, and so $R$ is an Artinian ring.

In the rest of the paper, we determine the number of edges in $\Gamma(R)$, denoted by $|\mathrm{E}(R)|$, in the case where $R$ is a finite direct product of fields. To this end, we first prove the following lemmas.

We denote by $\mathbb{I}(R)$ the number of nontrivial ideals of $R$, and by $r(R)$ the number of nontrivial ideals $I$ such that $I \nsubseteq \mathrm{Z}(I)$. Let $R=R_{1} \times \cdots \times R_{n}$ and $I$ be an ideal of $R$. We use $\pi_{i}(I)$ to denote the image of ideal $I$ of $R$ by the natural ring epimorphism $\pi_{i}: R \longrightarrow R_{i}$. Also, we use $\operatorname{Supp}(I)$ to denote the set of indices $i$ such that $\pi_{i}(I)=R_{i}$.

In the following lemma, we compute $r(R)$, where $R$ is an Artinian ring.

Lemma 3.5. Suppose that $R=R_{1} \times \cdots \times R_{n}$, where each $R_{i}$ is an Artinian local ring, for $i=1, \ldots, n$. Then $r(R)=2^{n}-2$.

Proof. Set

$$
\begin{aligned}
\Sigma & :=\{I \mid I \nsubseteq \mathrm{Z}(I), \text { for } I \notin 0, R\} \text { and } \\
\Sigma^{\prime} & :=\left\{I \mid \pi_{i}(I)=0 \text { or } R, \text { for } i=1, \ldots, n\right\} .
\end{aligned}
$$

Clearly $|\Sigma|=r(R)$ and $\left|\Sigma^{\prime}\right|=2^{n}-2$. So it is enough to show that $\Sigma=\Sigma^{\prime}$. To do so, 
suppose that $I \in \Sigma^{\prime}$. Consider the element $\mathbf{x}:=\left(x_{i}\right) \in R$, where

$$
x_{i}= \begin{cases}1 & \text { if } i \in \operatorname{Supp}(I) \\ 0 & \text { otherwise }\end{cases}
$$

Clearly $\mathbf{x}$ is $I$-regular. Hence $I \in \Sigma$ and $\Sigma^{\prime} \subseteq \Sigma$.

Now, if $\Sigma \nsubseteq \Sigma^{\prime}$, then there exists $I \in \Sigma$ such that $I \notin \Sigma^{\prime}$. Without loss of generality, we may assume that $\pi_{1}(I)$ is a nontrivial ideal of $R_{1}$. Since $I \in \Sigma$, there exists an $I$ regular element $\mathbf{y}=\left(y_{i}\right)$ with $y_{1} \notin \mathrm{Z}\left(I_{1}\right)$. Since $R_{1}$ is an Artinian local ring, any nonunit element in $R_{1}$ is a zero-divisor. Also, by Proposition 3.1, $\mathrm{Z}\left(I_{1}\right)=\mathrm{Z}\left(R_{1}\right)$, because $I_{1}$ is an isolated vertex in $\Gamma\left(R_{1}\right)$. Hence $y_{1} \notin \Gamma Z\left(R_{1}\right)$ which is impossible, and so for a maximal ideal $m$, we have $\operatorname{Ass}(R)=\{m\}$. Thus $\Sigma \subseteq \Sigma^{\prime}$ as desired.

Lemma 3.6. Assume that $F$ is a field and $R$ is an arbitrary ring. Then

$$
|\mathrm{E}(F \times R)|=3|\mathrm{E}(R)|+2 \mathbb{I}(R)+r(R) .
$$

Proof. For a subset $C$ in $\Gamma(F \times R)$, we denote the induced subgraph of $\Gamma(F \times R)$ with vertex set $C$ by $\Gamma(F \times R)[C]$. Now consider the collections $A:=\left\{F \times I_{i}\right\}_{i=1}^{\mathbb{I}(R)}$ and $B:=\left\{0 \times I_{i}\right\}_{i=1}^{\mathbb{I}(R)}$ of vertices in $\Gamma(F \times R)$. It is not hard to see that

$$
|\mathrm{E}(\Gamma(F \times R)[B])|=|\mathrm{E}(\Gamma(F \times R)[A])|=|\mathrm{E}(R)| .
$$

Also, clearly, for some ideals $I$ and $J$ of $R, F \times I \longrightarrow 0 \times J$ is an $\operatorname{arc}$ in $\Gamma(F \times R)[A \cup B]$ if and only if we have one of the following conditions:

(i) $\quad I=J$ and $I \nsubseteq \mathrm{Z}(J)$;

(ii) $I \neq J$ and $I \longrightarrow J$ in $\Gamma(R)$.

This implies that

$$
|\mathrm{E}(\Gamma(F \times R)[A \cup B])|=|\mathrm{E}(\Gamma(F \times R)[B])|+|\mathrm{E}(\Gamma(F \times R)[A])|+|\mathrm{E}(R)|+r(R) .
$$

Thus

$$
|\mathrm{E}(\Gamma(F \times R)[A \cup B])|=3|\mathrm{E}(R)|+r(R) .
$$

Now, since all vertices in $B$ are adjacent to $0 \times R$, the number of arcs from the vertex $0 \times R$ to the vertices in $B$ is equal to $\mathbb{I}(R)$. Similarly, the number of arcs from the vertices in $A$ to the vertex $F \times 0$ is equal to $\mathbb{I}(R)$. Also, there exists no edge between the vertices in $A$ and the vertices in $0 \times R$. Therefore, we can easily check that $|\mathrm{E}(F \times R)|=3|\mathrm{E}(R)|+2 \mathbb{I}(R)+r(R)$.

Theorem 3.7. Let $R=F_{1} \times \cdots \times F_{n}$, where $F_{i}$ is a field, for $i=1, \ldots, n$, where $n \geq 3$. Then $|\mathrm{E}(R)|=3^{n}-3\left(2^{n}-1\right)$.

Proof. Since $\mathbb{I}(R)=2^{n}-2$, by Lemma 3.5, we have $\mathbb{I}(R)=r(R)$. We use induction on $n$. Clearly, for $n=3, \Gamma(R)$ is isomorphic to $C_{6}$, and so the result holds. Now, suppose that $n=k+1$ and the result holds for smaller values of $n$. Assume that 
$R=F_{1} \times \cdots \times F_{k} \times F_{k+1}$. By the induction hypothesis, $\left|\mathrm{E}\left(F_{1} \times \cdots \times F_{k}\right)\right|=3^{k}-$ $3\left(2^{k}-1\right)$. Hence, by Lemma 3.6,

$$
\begin{aligned}
\left|\mathrm{E}\left(F_{1} \times \cdots \times F_{k} \times F_{k+1}\right)\right| & =3\left|\mathrm{E}\left(F_{1} \times \cdots \times F_{k}\right)\right|+2\left(2^{k}-2\right)+\left(2^{k}-2\right) \\
& =3\left(3^{k}-3\left(2^{k}-1\right)\right)+3\left(2^{k}-2\right) \\
& =3^{k+1}-3\left(2^{k+1}-1\right) .
\end{aligned}
$$

This concludes the proof.

REMARK 3.8. We now provide another proof of Theorem 3.7. For this purpose, suppose that $I(k)$ denotes the product

$$
F_{1} \times \cdots F_{k} \times \underbrace{0 \times \cdots \times 0}_{n-k \text { times }}
$$

for $k=1, \ldots, n-1$. First note that, for ideals $J_{i}$ of $F_{i}, I(k) \longrightarrow J_{1} \times \cdots \times J_{n}$ is an arc in $\Gamma(R)$, if and only if $J_{i}=0$ for all $i=k+1, \ldots, n$. Hence $\mathrm{d}^{+}(I(k))=2^{k}-2$. Similarly, $\mathrm{d}^{-}(I(k))=2^{n-k}-2$, and so $\operatorname{deg}(I(k))=\mathrm{d}^{+}+\mathrm{d}^{-}=2^{n-k}+2^{k}-4$.

Now, if $J$ is an ideal of $R$ with $|\operatorname{Supp}(J)|=k$, then, by using a suitable permutation on $\{1,2, \ldots, n\}$, it is easy to see that the degrees of vertex $J$ and $I(k)$ are equal in $\Gamma(R)$.

On the other hand, there are exactly $\left(\begin{array}{l}n \\ k\end{array}\right)$ ideals $J$ with $|\operatorname{Supp}(J)|=k$. Thus we have the equality

$$
2|\mathrm{E}(R)|=\sum_{k=1}^{n-1}\left(\begin{array}{l}
n \\
k
\end{array}\right)\left(2^{n-k}+2^{k}-4\right) .
$$

Now, by using the expansion of the function $f(x)=(1+x)^{n}-x^{n}-1$, we can easily see that $2|\mathrm{E}(R)|=2 f(2)-4 f(1)$, and so $|\mathrm{E}(R)|=3^{n}-3\left(2^{n}-1\right)$.

\section{Acknowledgement}

The authors are deeply grateful to the referee for a careful reading of this paper and helpful suggestions.

\section{References}

[1] M. Afkhami and K. Khashyarmanesh, 'The cozero-divisor graph of a commutative ring', Southeast Asian Bull. Math. 35 (2011), 753-762.

[2] D. F. Anderson, M. C. Axtell and J. A. Stickles, 'Zero-divisor graphs in commutative rings', in: Commutative Algebra, Noetherian and Non-Noetherian Perspectives, (eds. M. Fontana, S. E. Kabbaj, B. Olberding and I. Swanson) (Springer, New York, 2011), pp. 23-45.

[3] D. F. Anderson and P. S. Livingston, 'The zero-divisor graph of a commutative ring', J. Algebra 217 (1999), 434-447.

[4] I. Beck, 'Coloring of commutative rings', J. Algebra 116 (1998), 208-226.

[5] J. A Bondy and U. S. R. Murty, Graph Theory with Applications (American Elsevier, New York, 1976).

[6] F. R. DeMeyer and L. DeMeyer, 'Zero-divisor graphs of semigroups', J. Algebra 283 (2005), 190-198. 
[7] E. Estaji and K. Khashyarmanesh, 'The zero-divisor graph of a lattice', Results Math. 61 (2012), $1-11$.

[8] A. V. Kelarev, Graph Algebras and Automata (Marcel Dekker, New York, 2003).

[9] A. V. Kelarev and S. J. Quinn, 'A combinatorial property and power graphs of groups', Contrib. General Algebra 12 (2000), 229-235.

[10] A. V. Kelarev and S. J. Quinn, 'Directed graphs and combinatorial properties of semigroups', J. Algebra 251 (2002), 16-26.

[11] A. V. Kelarev, J. Ryan and J. Yearwood, 'Cayley graphs as classifiers for data mining: the influence of asymmetries', Discrete Math. 309 (2009), 5360-5369.

[12] C. H. Li and C. E. Praeger, 'On the isomorphism problem for finite Cayley graphs of bounded valency', European J. Combin. 20 (1999), 279-292.

[13] H. R. Maimani, M. Salimi, A. Sattari and S. Yassemi, 'Comaximal graph of commutative rings', J. Algebra 319 (2008), 1801-1808.

[14] M. J. Nikmehr and F. Shaveisi, 'The regular digraph of ideals of a commutative ring', Acta Math. Hunger. 134 (2012), 516-528.

[15] C. E. Praeger, 'Finite transitive permutation groups and finite vertex-transitive graphs', in: Graph Symmetry: Algebraic Methods and Applications (Kluwer, Dordrecht, 1997), pp. 277-318.

[16] P. K. Sharma and S. M. Bhatwadekar, 'A note on graphical representation of rings', J. Algebra 176 (1995), 124-127.

[17] R. Y. Sharp, Steps in Commutative Algebra, 2nd edn, London Mathematical Society Student Texts, 51 (Cambridge University Press, Cambridge, 2000).

[18] A. Thomson and S. Zhou, 'Gossiping and routing in undirected triple-loop networks', Networks 55 (2010), 341-349.

[19] H. J. Wang, 'Graphs associated to co-maximal ideals of commutative rings', J. Algebra 320 (2008), 2917-2933.

[20] S. Zhou, 'A class of arc-transitive Cayley graphs as models for interconnection networks', SIAM J. Discrete Math. 23 (2009), 694-714.

M. AFKHAMI, Department of Mathematics, University of Neyshabur,

PO Box 91136-899, Neyshabur, Iran

e-mail: mojgan.afkhami@yahoo.com

M. KARIMI, Department of Pure Mathematics, Ferdowsi University of Mashhad,

PO Box 1159-91775, Mashhad, Iran

e-mail: karimimth@yahoo.com

K. KHASHYARMANESH, Department of Pure Mathematics, Ferdowsi University of Mashhad, PO Box 1159-91775, Mashhad, Iran

e-mail: khashyar@ipm.ir 